Revue des patrimoines

15 | 2011

Le patrimoine des guides : lectures de l'espace urbain européen

\title{
Les guides comme corpus de la connaissance urbaine
}

\section{Marcel Roncayolo}

\section{(2) OpenEdition Journals}

Édition électronique

URL : http://journals.openedition.org/insitu/559

DOI : 10.4000/insitu.559

ISSN : 1630-7305

Éditeur

Ministère de la culture

Référence électronique

Marcel Roncayolo, "Les guides comme corpus de la connaissance urbaine », In Situ [En ligne],

15 | 2011, mis en ligne le 29 juin 2011, consulté le 20 avril 2019. URL : http://journals.openedition.org/ insitu/559 ; DOI : 10.4000/insitu.559

Ce document a été généré automatiquement le 20 avril 2019.

\section{cc) (†)}

In Situ Revues des patrimoines est mis à disposition selon les termes de la licence Creative Commons Attribution - Pas d'Utilisation Commerciale - Pas de Modification 4.0 International. 


\title{
Les guides comme corpus de la connaissance urbaine
}

\author{
Marcel Roncayolo
}

\section{NOTE DE L'ÉDITEUR}

Entretien avec Marcel Roncayolo

Réalisé par Joanne Vajda le 3 juin 2009

Retranscription : Solange Pawou-Molu et Rosine Feferman

Relectures : Rosine Feferman

JV : Avez-vous déjà utilisé des guides de voyage et de tourisme pour vos propres recherches? De quelle façon vous en êtes-vous servi?

1 MR: Alors, premier point : les guides ne sont pas uniquement de voyage ou de grand voyage. Le rôle du guide, c'est de guider aussi bien l'habitant que le touriste, que le visiteur ; et d'ailleurs là, c'est, non pas une des ambiguïtés, mais l'un des recouvrements, et l'une des rencontres qui n'est pas sans intérêt et dont nous verrons l'importance ensuite. Mais prenons plutôt la question : comment ai-je été amené à me servir des guides pour connaître les villes ? Pas seulement les villes d'ailleurs. Pour tout dire, ma première rencontre sérieuse avec les guides, je dis bien sérieuse, de telle manière que cette lecture ait une dimension autre que la simple connaissance du lieu que je visitais ou dans lequel je me déplaçais, a eu lieu lorsque j'ai un petit peu - parce qu'on ne le préparait pas à proprement parler - préparé le concours général de géographie en 1942...

$\mathrm{JV}$ : Avez-vous été récompensé ?

MR : Oui, cela m'a valu de recevoir le premier prix.

Finalement, par rapport aux livres de géographie, le seul moyen d'avoir une autre vision, à la fois plus précise et plus en mouvement, a été de lire les guides. Par mouvement, je n'entends pas seulement le mouvement spatial, mais le mouvement du temps, le mouvement historique. Je me souviens être allé voir un spectacle d'opérette avec mes parents. C'était $L a$ fille du régiment. J'avais apporté un guide qui portait sur une région qui 
n'est pas tombée finalement au concours général. C'était le guide de la Lorraine. Et, pendant l'entracte, je me suis plongé dedans, avec empressement, car j'étais curieux de savoir comment le fameux problème des cuestas - qui est présenté comme un modèle et un passe-partout dans les livres de géographie - était représenté pour la Lorraine. C'est l'utilisation des guides qui m'a aidé à devenir géographe, parce que j'étais historien et géographe à la fois, sans trancher vraiment. Ce qui m'a fait faire du progrès dans la géographie, c'est la lecture des guides, parce que précisément ceux-ci ne niaient pas l'histoire. Au contraire, ils la replaçaient dans un contexte géographique. C'est pourquoi je reste un lecteur de guides, des bons voire des mauvais !

\section{JV : Quelle légitimité peut avoir ce type de source pour l'écriture de l'histoire urbaine?}

MR : Cette légitimité est d'abord une légitimité pratique, finalement. Le guide permet le contact avec le réel. Et je crois que découvrir le réel est une des démarches essentielles dans ma discipline. Qu'est-ce que j'entends par réel ? Et c'est là que la légitimité du guide devient plus complexe, ce ne sont pas seulement les choses telles qu'elles sont, parce que personne ne voit les choses telles qu'elles sont. On voit toujours les choses tel que l'on est placé dans le temps, c'est-à-dire avec le souvenir que l'on peut en avoir, sa culture et ses préjugés. Cela dépend aussi des positions, des points de vue. Donc le guide est précisément ce qui accorde une certaine légitimité à une subjectivité un peu trop inquiétante parce qu'on ne sait pas, après tout, si on regarde bien, si on voit bien les choses. Il y a un phénomène presque d'étiquette dans le guide qui vous permet ce rapport avec un réel un peu plus objectif, qui permet de créer l'objet, qui donne l'impression de participer à sa création. Mais cela dit, c'est un objet qui lui-même est subjectif, parce que l'intérêt du guide, aussi, est que le réel n'est pas seulement le réel des choses. C'est le réel de la manière dont on pense les choses. Cette double entrée du guide me semble extrêmement riche. La collection des guides dans le temps, par exemple, vous permet de voir comment changent les points de vue sur la ville et sur les éléments de la ville, sur les itinéraires à suivre. Autrement dit, la véritable légitimité du guide vient du fait qu'il offre une lecture de la pratique historique de la ville.

5 Le guide est d'une grande richesse car il donne une vision de la ville en rapport avec la mémoire, selon les dates et les traces différentes qu'il évoque. On retrouve un peu dans les guides la dimension du livre de Julien Gracq sur Nantes ${ }^{1}$.

JV: Parlons de la relation entre la connaissance scientifique et les guides de voyage. Élisée Reclus, géographe, rédige plusieurs Guides Joanne. Comment sa connaissance du territoire et son regard de géographe contribuent-ils à la diffusion d'une vision de l'espace? Et quelle est précisément sa vision de l'espace à travers ses publications?

MR : Chez Reclus, on ne retrouve pas une vision de l'espace construit comme approche abstraite et explicative des choses. Ce n'est pas Christaller. Fort heureusement. Cela m'a sauvé, si j'ose dire, de Christaller $^{2}$, dont le modèle est un modèle géométrique d'explication du réseau urbain dans ses hiérarchies par le jeu de la distance-temps (à supposer qu'elle ait toujours la même valeur !).

7 Reclus ${ }^{3}$ est un type extraordinaire qui s'est intéressé aux guides, bien que ce ne soit pas lié à sa formation. Il a plutôt une formation théologique, mais il s'est intéressé aux guides par sa vie, par les accidents de sa vie. Reclus est un des Français qui a le moins résidé en France. Cela n'est pas dû à son simple désir de voyager, mais au fait qu'il était interdit de séjour en France, étant donné que c'était un «mal-pensant ». Après chaque révolution, il passait un certain temps à l'extérieur. 
8 La légitimité de Reclus vient surtout du fait que la culture qu'il avait, forgée autour des questions de compréhension de la nature qui était très dix-neuvième siècle - liée aux théories de Darwin qui ont révolutionné la biologie - et sa recherche, à travers laquelle il s'intéressait à la manière dont les choses se tressaient dans l'histoire, lui ont permis de faire lui-même l'expérience de pays étrangers, voire éloignés. Il a surtout vécu en Angleterre, en Suisse et en Italie, mais en même temps, il s'était créé un véritable réseau.

On remarque cette qualité de Reclus encore plus dans le dictionnaire Larousse, frère jumeau des Guides Joanne, en particulier par les personnalités qu'il sait solliciter pour parler de tel territoire et qui sont des diplomates, des voyageurs, etc. On ne pratiquait pas beaucoup d'interviews à l'époque, enfin on publiait encore moins, mais je suis persuadé que Reclus était un intervieweur de premier ordre. Autrement dit, il a constitué une sorte de mémoire de tout ce qui s'écrivait sur l'extérieur, fondé sur un réseau d'informations et sur l'étude critique de ce qu'il connaissait lui-même directement. Il y a cette grande qualité chez Reclus, qu'il ne s'est pas enfermé dans son local. Mais en même temps il a traité les particularités du local. Il ne s'est pas enfermé non plus dans une conception très abstraite de la géographie qui consisterait à essayer de trouver la même chose partout. Il est arrivé à composer des vues diverses.

En ce qui me concerne, j'ai travaillé sur Marseille en particulier, dans des conditions assez redoutables, puisque c'était ma ville de naissance et donc il y avait toujours une sorte de rapport non scientifique avec l'objet que j'étudiais. Reclus m'a appris beaucoup de choses justement sur cette ville que je connaissais. Par certains côtés il l'a comprise en son temps mieux que moi, ou tout au moins il a mis en valeur des éléments que j'aurais eu plus de difficultés à distinguer après coup. Reclus est en grande partie un observateur. Mais c'est un observateur cultivé, qui dispose de beaucoup de références scientifiques. C'est un mondialiste si vous voulez, ce qui est très important pour la géographie. C'est le local, c'est-à-dire le singulier à travers le voyage, le général, qui me semble avoir été la grande vision de Reclus. Et donc sa légitimité en réalité. En même temps, il savait passer à l'idée générale mais justement sans se contenter d'une sorte d'approche systématique. C'est aussi un critique de l'idée de système.

11 Pour moi, Reclus, est en soi le géographe idéal, avec le décalage de temps bien entendu qui s'impose. Sa légitimité est acquise historiquement et à l'épreuve. Reclus a toujours évité de tomber dans l'hagiographie. Et il m'arrive de m'irriter parce que les guides que je trouve dans des pays étrangers ne sont pas assez inspirés de sa démarche. Il y a un peu du franco-français dans mon admiration pour Reclus!

$\mathrm{J}$ : Comment, selon vous, la recherche à partir des guides de voyage et de tourisme peut-elle contribuer à l'étude de la construction des espaces urbains en Europe?

MR: Dans le fond, je vous ai donné l'idée générale parce qu'il faut avoir à la fois une démarche analytique, c'est-à-dire qu'il faut voir ce qui se distingue, et il faut voir dans quelle mesure les villes ne sont pas des morceaux juxtaposés qui n'ont rien à se dire. Une ville, ça fonctionne bien ou mal, mais avec toute sa diversité. J'ai tendance à placer le point de départ du géographe, ou de l'observateur, dans la notion de simultanéité. Ce sont des choses simultanées que l'on voit. Et il s'agit de savoir si elles ont des rapports entre elles ou pas, cela peut se produire, et donc quel est le type d'articulation qui en même temps ne peut s'expliquer que parce que ce sont des temporalités très différentes qui se retrouvent dans la simultanéité, soit par l'origine, soit par le déroulement, soit par le degré de vieillissement, soit par l'interprétation même que l'on donne, qui n'est pas forcément celle de départ. C'est cette sorte de tension entre la simultanéité et la non 
synchronie des choses qui me semble la démarche géographique intéressante à suivre pour les villes principales. C'est d'ailleurs finalement la démarche des géographes physiciens dès qu'ils se rapprochent un peu de la géologie. Cela n'a rien d'exceptionnel, mais il faut le concevoir pour la ville, ce qui n'a pas été perçu d'emblée. C'est-à-dire qu'il faut qu'il y ait sans cesse analyse et synthèse. L'analyse seule risque d'être insuffisante, car on risque de manquer une étape ou une ancienneté réelle que l'on ne perçoit pas. Le savant se soucie de voir comment les choses se sont mises en rapport entre elles. Je crois beaucoup au philosophe Alain. Alors comme vous parlez d'espace, Alain et moi, nous nous méfiions de l'espace parce que l'espace c'est moins une réalité, que le rapport entre les choses, et entre le sujet et les choses. Un rapport triangulaire donc.

JV: À votre avis, une approche pluridisciplinaire à partir de l'étude des guides, peut-elle renouveler la connaissance que nous avons déjà de la ville ?

13 MR : Les guides, c'est non seulement de la géographie au sens premier du terme, ce sont aussi des sites qualifiés. Il y a donc déjà de l'interprétation dans les guides, car les choses ne sont pas situées sur le même plan.

14 J'ai eu du mal avec certaines personnes que j'apprécie, en particulier avec les architectes, dans la mesure où, souvent, ils n'ont pas fait de géologie. Ils ne comprennent pas immédiatement le sens dans lequel j'emploie le mot «strates». Or, dans les guides, on retrouve tout le problème de la stratification. Sur les cartes géologiques, il y a l'histoire stratifiée, chaque période étant qualifiée par une couleur sur la carte et aussi par la nature du dépôt, calcaire coquillier, etc. de telle époque. C'est un entassement d'époques, de la plus ancienne jusqu'à la plus récente. Le problème ensuite est de savoir comment, ces différents éléments affleurent ou jouent un rôle dans le paysage. Autrement dit, il ne s'agit pas simplement de creuser pour trouver le plus ancien. Le plus ancien est souvent en surface. C'est une géologie extrêmement mouvementée où le plus ancien peut se superposer au plus récent. On retrouve dans les guides la différence qu'il y a entre ce qu'on appelle en géologie les affleurements et la stratification historique, au sens de l'histoire géologique. C'est en travaillant que j'ai compris l'importance de la géographie physique, que je n'aimais pas beaucoup. Elle permet une approche pluridisciplinaire. Grâce à elle j'ai pu bien comprendre la géologie et plus précisément les ambiguïtés de la géologie et de l'âge, de l'affleurement, pour l'appliquer ensuite à la ville. La différence est que pour la géologie on voit une photo d'un paysage. Pour la ville, qui est l'endroit où l'on vit, la simultanéité a tout de même une présence réaliste, ce n'est pas simplement un objet à regarder, à contempler.

JV : Et comment les guides permettent-ils justement de saisir la complexité d'un territoire, d'une ville?

15 MR : Les géographes actuels ont tendance à dire que la géographie a été renouvelée par la notion d'espace-temps utilisée par les physiciens qui estiment que l'observateur et les déplacements de l'observateur modifient l'objet. La notion d'espace-temps, me semble quelque chose de vraiment fondamental pour la ville et pour la campagne aussi.

16 Je crois que la géographie c'est finalement cela. Et j'en reviens à la simultanéité : dans un paysage, à un moment déterminé, vous voyez à la fois l'espace, c'est-à-dire la distance entre les choses et vous vous dites bien que tout cela ne date pas exactement de la même période. Personnellement, je n'ai jamais pu dissocier une démarche géographique d'une démarche historique. Il fallait que je qualifie, que j'arrive à voir l'origine. Alors, évidemment, il faut se fixer des points de départ. Si l'on remonte à la géologie, justement, 
on ne s'en sort plus, mais il faut se fixer des limites. Je me suis attaché surtout aux apports du XIX siècle et du premier $\mathrm{XX}^{\mathrm{e}}$ siècle, $\mathrm{j}$ 'ai essayé de décortiquer les éléments selon le moment et la manière dont ils entraient en scène, la manière dont ils étaient interprétés quand ils entraient en scène, afin de voir comment on pouvait modifier cette interprétation. C'est ma démarche. Je ne dis pas qu'il n'y a que celle-ci qui est bonne, mais je ne peux pas travailler autrement.

$J V$ : Comment peut-on articuler la réalité d'une ville et les représentations qu'en donnent les guides?

MR: Ce qui est intéressant dans les guides, c'est qu'il y a une stratification des représentations. Ils inscrivent à un moment noir sur blanc des représentations, mais ces représentations, ce ne sont pas les guides qui les apportent.

Les guides sont dans une situation très curieuse, c'est ce qui me passionne dans leur cas. Ils sont la représentation de ce qui est accepté par l'habitant. Il faut une reconnaissance de l'habitant. Et il faut aussi captiver l'intérêt du visiteur. Je trouve donc que le guide est par définition une médiation de représentations. Il ne faut pas décevoir le visiteur, alors il faut un peu lui dire ce qu'il s'attend à voir ! Quitte même à le choquer, à jouer sur des contrastes. Le guide est un médiateur de représentations acquises, de représentations des uns et des autres, des observateurs différents, et de représentations en mouvement. C'està-dire que cela peut changer. Je prends toujours l'exemple pour Marseille de la fameuse Vieille Charité, qui est considérée maintenant comme le monument par excellence de Marseille, qui est très beau d'ailleurs. C'est une création du XVII ${ }^{e}$ siècle, achevée au XVIII ${ }^{e}$ siècle. L'intérêt porté par les guides et que l'on fait donc porter sur le visiteur, même sur l'habitant, suit l'histoire de cet ancien hospice. Au XVIII ${ }^{\mathrm{e}}$ siècle on se payait de magnifiques constructions pour ce type de programme. Le bâtiment n'a cessé de se dégrader, faute d'entretien, notamment après la Révolution. Il se trouve que ma grandmère était née dans les vieux quartiers, à côté de la Vieille Charité, sur la place des Moulins qui dominait le reste. Le reste, c'était le bas, le condamnable. La Vieille Charité faisait partie du condamnable. Et on ne m'a jamais emmené voir ce bâtiment dans mon enfance. On me faisait faire le tour des vieux quartiers, des extérieurs, si j'ose dire, mais il n'était pas question de me faire connaître les vieux quartiers qui avaient été pourtant pour une partie de ma famille un lieu de naissance et de vie. Lorsque j'avais 14 ou 15 ans, quand j'ai commencé à me balader - on sortait seul tardivement à cette époque - je me suis dit qu'il fallait que j'aille voir ce que c'était. Je suis arrivé sur une Vieille Charité qui était vert-de-grisée au possible, avec des pieux plantés dans les façades pour empêcher que les gens ne s'échappent par les fenêtres. C'était en plus sous l'Occupation. C'était épouvantable! C'est ainsi que je me suis rendu compte que la Vieille Charité, durant tout le XIX siècle, avait été un long glissement de l'être vers le non-être.

On a failli la détruire pour y construire un bel ensemble de logements sociaux et finalement on l'a sauvée. Maintenant c'est devenu le chef d'œuvre baroque de Marseille.

Et, les guides, même s'ils ne sont pas toujours remis à jour, s'ils ont parfois un peu de retard, sont intéressants par leur date, dans la mesure où l'on retrouve une cotation, comme à la Bourse. Il y a un côté boursier dans le guide, sur la valeur que l'on attache aux choses. Tantôt c'est le plus vieux qui prend de la valeur, tantôt c'est le plus jeune. Il faut le lire comme ça le guide. C'est donc une représentation hiérarchisée.

21 En soi, je crois que le guide est un objet de travail. Ce n'est pas seulement le moyen d'accéder à un objet urbain, avec beaucoup de nuances, et en faisant valoir beaucoup 
d'éléments intellectuels et sentimentaux de valorisation, mais c'est en même temps, en soi, un objet qu'il faut étudier comme un document, un document de représentation. Mais il faut l'objectiver plus précisément. Et cela est très profitable pour la science. Les hommes du dix-neuvième siècle le sentaient d'ailleurs très bien, sans être des spécialistes de guides. C'étaient des gens cultivés. Par certains côtés, le meilleur guide critique de la Grèce du XIX ${ }^{e}$ siècle est dû à un littérateur, normalien qui écrivait des romans, et des romans quelquefois plutôt goguenards, mais il a fait un guide sur la Grèce qui était splendide ${ }^{4}$

Alors, en même temps vous voyez très bien le dédoublement : c'est toujours le monsieur qui regarde le monsieur, comme dans les publicités pour la peinture Ripolin. Pour les guides, c'est un peu la même chose, il faut critiquer la critique. Je ne comprends pas une ville, maintenant, que j'essaie de comprendre sérieusement, sans lire les guides successifs. Et en particulier pour l'Italie qui est un des pays que je fréquente le plus, je possède une série de guides qui est assez intéressante de ce point de vue.

$\mathrm{JV}$ : Vous utilisez plusieurs collections en même temps?

MR : Oui. Je dirais que ce qui m'importe c'est la date et la maison d'édition. Chaque maison a ses interprétations qui durent, ne serait-ce que parce qu'on réédite souvent et que par conséquent on ne fait pas toujours des mises à jour. Mais au total il y a quand même des évolutions dans chaque guide.

Et il y a maintenant le nouveau principe du guide. Alors, peut-être allons-nous trouver une autre dimension du guide qui redevient guide et non plus document historique d'abord. C'est sa légitimité pour guider les pas de quelqu'un. C'est le guide comme inventeur d'itinéraires. C'est un aspect fondamental car vous connaissez surtout une ville par les itinéraires qui y sont proposés. Il faut reprendre votre liberté un petit peu par rapport à cette facilité de suivre les itinéraires, mais justement, cela est aussi fondamental. Cette fois-ci ce n'est pas l'effet de la représentation, mais cela commande la représentation. Moi qui suis, sur le plan scientifique, un analyste de la ville, je suis un mordu de l'itinéraire. Une ville c'est avant tout des itinéraires. Cela va assez loin parce que je sors d'un colloque qui s'appelait Lieux et liens, et ce qui m'intéresse dans la ville c'est le lien entre les lieux beaucoup plus que le lieu créateur de liens ou les liens créateurs de lieux, c'est le lien entre les lieux. Et moi-même, puisque j'ai vécu longtemps au gré de l'expérience que me transmettaient mes parents, je suis bourré d'itinéraires qui ne sont pas forcément les plus logiques et les plus reconnus mais qui sont les miens. J'ai mes itinéraires dans la ville. Et je crois que la force du guide justement, c'est de ne pas enfermer les choses dans des lieux, dans un lieu, c'est de savoir - et tous les guides ne réussissent pas aussi bien - non pas juxtaposer mais enchâner, même quand c'est au prix d'une pirouette historique, qu'il y a rupture réelle. Il faut la sentir, la rupture réelle, et c'est d'ailleurs le charme des itinéraires en général. C'est une vertu du guide dont on n'a pas encore parlé. Ce n'est pas simplement un guide des pas - c'est quand même pratique quand on veut aller d'un point à un autre - cela fait découvrir cette autre réalité profonde de la ville, et plus profonde que chaque lieu pris séparément, qui est l'enchaînement des lieux.

JV: Et pourtant, peu de guides parlent des liens justement. En général, ils vous envoient d'un objectif à un autre..

MR: Il y a quand même des itinéraires. Les guides classiques étaient des itinéraires. Certains guides récents ont fait un compendium d'articles historiques qui sont fort intéressants, mais où cette notion d'enchaînements physiques se perd. Or, le guide, c'est 
ce qu'il y a de mieux pour le piéton. Personnellement, je ne connais une ville que lorsque je suis piéton. Il n'y a rien à faire, il suffit que j'entre dans une voiture pour ne plus savoir justement quel est le rapport entre les lieux.

J'utilise les transports quand il faut les utiliser, mais vraiment je n'entre dans une ville que par les pieds. Et en faisant moi-même mes itinéraires. Alors, j'ai les itinéraires proposés par le guide et justement, l'art, c'est de jouer avec ceux-ci.

Le guide est vraiment fondamental. Il est l'élément qui me sert à la fois de guide, c'est le cas de le dire, et de repoussoir, de mise en question. Et en même temps, cela va de la jouissance de la ville enfin de la reconnaissance de la ville, mais je crois que cela conduit aussi à une démarche historique, à une démarche scientifique sur la ville. Je crois d'ailleurs que, pour la ville, on ne peut pas couper la pratique banale de la pratique scientifique. Et que là aussi c'est la médiation qu'il faut trouver. Ce n'est pas si commode d'ailleurs.

JV: Et par rapport à cette question de la construction des itinéraires, que pensez-vous de la cartographie et des plans qui sont publiés dans les guides?

MR : La cartographie est un mal nécessaire, parce que la cartographie a quand même une utilité majeure. Mais la cartographie est une abstraction parce que vous ne marquez pas tout, ce n'est pas une photographie, et même sur la photographie, il y a des choses qu'on voit et des choses qu'on ne voit pas. Mais je dirais que la cartographie est plus honnête que la photographie dans un certain sens parce qu'elle n'a pas la volonté de représenter la réalité tout entière et dans toute sa diversité. Il y a un choix, un choix de ce que l'on représente. Qu'elle soit réaliste ou pas, c'est un phénomène de signes. D'autre part, la cartographie est rigoureusement liée au rapport que l'on établit entre ce que l'on représente et la manière dont on le représente, donc à l'échelle. Et toute cartographie est dépendante de l'échelle que l'on choisit. Il ne faut pas subir passivement l'échelle, mais au contraire connaître les contraintes imposées par l'échelle. La cartographie utilise depuis un certain temps les vues satellitaires. Personnellement, j'ai commencé à les utiliser très tard systématiquement, parce que généralement on me proposait des vues par satellite selon une échelle choisie préalablement. Récemment lorsque j'ai travaillé sur Nanterre, au contraire, j'ai utilisé le zoom à partir de la vue satellitaire, c'est-à-dire en partant de Sirius et en descendant au plus petit et au plus concret possible. Chose d'ailleurs qui est remarquablement faite dans un film, qui m'a servi de méthodologie de ce point de vue, et qui s'appelle American Beauty ${ }^{5}$. Ce n'est pas le premier, me semble-t-il, mais c'est le premier qui s'en soit servi vraiment pour donner un sens à cette descente. L'intérêt du zoom, est que vous n'avez pas une échelle pré indiquée, sur laquelle vous êtes obligé de travailler, parce que c'est ainsi que le document se présente. Vous choisissez, subjectivement bien entendu, l'échelle qui vous parle. Et vous pouvez trouver d'ailleurs des problématiques très différentes en changeant d'étage, si j'ose dire. Je me suis régalé en travaillant ainsi! Je n'ai moi-même compris Nanterre qu'en zoomant, zoomant, zoomant.

Donc la cartographie c'est le mal nécessaire parce que lorsque vous êtes obligé de reproduire des zooms cela n'est pas facile. Et ce n'est pas sûr que cela produise le même effet. La cartographie vous sert à choisir une échelle, à mettre sur cette échelle les signes que vous pensez compatibles avec l'échelle, d'une part, et que vous pensez compatibles avec ce que vous voulez exprimer d'autre part. Donc il n'y a rien de plus travaillé que la cartographie, avec tous les risques que cela peut représenter par ailleurs. La cartographie est un travail intellectuel et c'est d'ailleurs la raison pour laquelle je suis entré, non pas 
en conflit, mais en désaccord critique, avec les cartographes qui se voulaient scientifiques et qui avaient comme ambition de projeter des statistiques dans l'espace. Pour moi c'est artificiel. Je ne projette que ce que je comprends, que les signes que je contrôle entièrement et non pas des chiffres dont je ne connais pas exactement l'origine, la critique, la valeur relative. Je crois que la cartographie automatique peut servir celui qui contrôle tous les éléments qui y participent et ne peut pas servir uniquement par sa technique de révélateur d'une réalité spatiale.

JV: Que pensez-vous de la question du découpage territorial ? Je sais que c'est un domaine où vous hésitez à vous exprimer très librement...

MR : Ah! D'abord, je vous ai dit que je n'aimais pas le terme « espace " parce que c'est une abstraction l'espace, on n'est pas dans le réel. Et je reprends volontiers le philosophe Alain - qui a été ma lecture de chevet - qui considère que les choses sont dans l'espace et que l'espace n'est pas dans les choses. Ce n'est pas une qualité des choses, mais les choses sont dans l'espace. C'est assez Kantien finalement comme référence initiale, et je me rallie volontiers à ce point de vue. En revanche j'adore la notion de territoire, après avoir d'ailleurs suivi moi-même une courbe. Je m'étais emparé du mot « espace » pour lutter contre l'idée que le découpage régional avait une réalité pérenne... La notion de région par exemple. Je me méfiais un peu de ces créations de choses dont, après tout, on peut toujours se demander dans quelle mesure elles existent ou pas, ou quel est leur rôle et leur durée historique, leur cohérence et l'emplacement réel qu'elles occupent. Alors j'avais pris " espace», qui me semblait plus neutre et ouvrait à toutes les utilisations et usages possibles. Mais ensuite, je me suis aperçu que le terme "espace », menait vers la géométrie et que cela se réduisait souvent à des géométries un peu simplistes type Christaller. Ce n'est pas faux mais, à mon avis, ce qui est intéressant, ce n'est pas de se rapporter à ce qui relève de l'évidence, ce qui est intéressant pour la géographie, c'est de voir dans quelle mesure ce terme est mal adapté. C'est ainsi que j'en suis venu à la notion de territoire, notion chérie qui reprend un peu les termes d'Alain. Le territoire c'est le rapport entre les choses et entre moi et les choses. C'est un rapport. Et c'est un rapport qui, par conséquent, supporte toutes les transformations qu'un rapport peut subir.

31 Se pose tout de même le problème du sujet, il y a des sujets individuels et des sujets collectifs. Un territoire, peut être conçu par un sujet collectif. Et vous-même pouvez avoir votre interprétation individuelle, détachée ou non de ce sujet collectif. Donc un territoire, c'est forcément problématique. Par exemple, prenons la notion de quartier qui est souvent développée pour l'analyse de la ville. Le quartier évidemment existe par certains côtés, il y a des liens qui s'expriment par le quartier, mais il me semble qu'enfermer le quartier, croire que la ville est une sorte de collection de quartiers juxtaposés, cela n'a qu'un sens appauvri. Grâce aux débats que j'ai eus avec des paysagistes, je me suis rendu compte qu'un territoire n'a de valeur que dans la mesure où il est en même temps perspective. Et un quartier, par exemple, n'a de sens que dans la mesure où il est lié à d'autres quartiers et donc c'est cette perspective qui est aussi intéressante... Il y a des lieux en effet fermés sur eux-mêmes, et malheureusement on en connaît beaucoup maintenant, sans perspective, et c'est au contraire la perspective qui fait le quartier ou l'intérêt du quartier. Cela me semble fondamental car étroitement lié à l'idée de territorialité, contre la géométrie de l'espace et contre l'être de raison que sont très souvent la région, le quartier etc. comme s'il s'agissait d'entités intouchables.

Cela rejoint la question de l'itinéraire. Pour moi la ville, ce sont les relations entre villes, les relations à l'intérieur de la ville, les relations même dans un quartier, ce sont des 
itinéraires finalement. Quand vous allez acheter des cigarettes, ou autre chose, ce sont des micro-itinéraires. L'école, la carte scolaire, c'est une manière de mettre en question la notion de quartiers fermés. Mais en réalité ce sont des territoires constitués en fonction de l'idée que les parents se font de la formation de leurs enfants, et qui constituent un territoire scolaire à leur manière dans la mesure où ils échappent, et ce n'est pas très difficile, aux contraintes légales de la carte scolaire. Alors, la notion de «territoire ", me ramène vraiment à de la géographie concrète, mais en même temps une géographie concrète qui est l'effet de représentations, de rapports. Maintenant, c'est vraiment le mot que j'emploie en priorité.

Les découpages, il n'y a rien de plus historique, ce n'est pas un problème d'histoire au sens événementiel du terme forcément, mais cela se déroule dans le temps. Le territoire, ça bouge, et ses limites aussi. Et, en particulier, je ne crois pas que l'on puisse agir sérieusement dans une pensée qui serait totalement figée. Je dis volontiers, et cela intéresse beaucoup les architectes, que ce n'est pas le découpage, c'est-à-dire le territoire pré-jugé, qui doit déterminer le projet, c'est le projet qui doit déterminer le territoire sur lequel il porte. Pour moi, c'est devenu un «delenda est Carthago ».

Mon ouvrage sur Nanterre s'appelle, avec une perfidie totale, une ambiguïté totale, Territoires en partage, la notion de partage étant bien entendu la plus ambiguë parce que cela peut être la division totale, le partage, ou le partage au sens communion du terme, le repas en commun. Voilà ! C'est toute la question du territoire.

Lorsque j'étais directeur adjoint de l'École Normale, j'ai fait un séminaire "Territoire ». Toutes les disciplines étaient représentées. Et l'on s'est battu autour de l'idée de territoire qui n'était pas encore totalement placée au cœur de la géographie. C'est le projet quand même qui a amené le territoire. Et j'ai écrit un livre. Les livres sont toujours très tardifs par rapport au moment où je les écris. À l'origine ce sont les articles Citta et Territorio publiés dans l'encyclopédie italienne Einaudi. Ce sont les Italiens qui en ont eu la primeur, en particulier les architectes italiens qui m'ont connu avant les Français. Cela a donné la version française enrichie, La ville et ses territoires ${ }^{6}$. Depuis, j'ai l'impression que le mot territoire a tout envahi! Alors je ne sais pas si je n'ai été que l'effet, un peu comme Halbwachs parlait des spéculateurs ${ }^{7}$, on prend une petite avance mais en réalité on ne fait qu'exprimer une attente commune, ou si j'ai joué un rôle beaucoup plus positif, mais c'est vrai que depuis on ne parle plus que de territoire. En même temps, cela mettait en question la fermeture de la ville comme objet géographique par rapport à ce qu'on appelle la campagne, c'est aussi un point critique. La ville et ses territoires, c'est que la ville est territoire, ensemble de territoires, et en même temps elle a des territoires, elle agit sur des territoires, elle traverse des territoires. Le territoire est à différentes dimensions, à différents usages. Le territoire est à la fois un nœud et un ensemble d'itinéraires. Et voilà comment "territoire" nous renvoie finalement à "guide». Le guide, c'est l'introduction à un territoire, bonne ou mauvaise, avec justement cette notion fondamentale de rapport, de médiation qui est notre situation anthropologique par rapport à l'espace évidemment, et qui change par conséquent. 


\section{BIBLIOGRAPHIE}

(Principaux ouvrages) :

RONCAYOLO, Marcel. La Ville et ses territoires. Paris : Gallimard, 1990, 278 p.

RONCAYOLO, Marcel. Villes et civilisation urbaine, $\mathrm{XVIII}^{\mathrm{e}}-\mathrm{XX}^{\mathrm{e}}$ siècles, anthologie réalisée avec Thierry Paquot. Paris : Larousse, 1992, 687 p.

RONCAYOLO, Marcel. Les Grammaires d'une ville. Essai sur la genèse des structures urbaines à Marseille. Paris : EHESS, 1996, 507 p.

RONCAYOLO, Marcel (dir.). Histoire de la France urbaine, t. 5, La ville aujourd'hui. Mutations urbaines, décentralisation et crise du citadin. Paris : Le Seuil, 2001, 898 p.

RONCAYOLO, Marcel. Lectures de villes : formes et temps. Marseille : Éd. Parenthèses, 2002, 386 p.

RONCAYOLO, Marcel. Territoires en partage. Nanterre, Seine-Arche, en recherche d'identité (s). Marseille : Éditions Parenthèses, 2007, 192 p.

\section{NOTES}

1. - GRACQ, Julien. La forme d'une ville. Paris : Corti, 1985.

2. - Walter Christaller (1893-1933), géographe allemand, auteur de théories ayant dissocié l'analyse spatiale et la géographie, dont les thèses avaient déjà été pressenties par Jean Reynaud (1806-1863), ingénieur saint-simonien, sous-secrétaire d'État à l'Instruction Publique sous Carnot en 1848.

3. - Élisée Reclus (1830-1905), géographe français, fils de pasteur protestant.

4. - ABOUT, Edmond. La Grèce contemporaine. Paris, Hachette, 1854.

5. - Film américain de 1999. Réalisation Sam Mendes. Avec Kevin Spacey, Annette Bening, Thora Birch.

6. - RONCAYOLO, Marcel. La ville et ses territoires. Paris : Gallimard, 1990, réédité en 1997 et en 2005.

7. - HALBWACHS, Maurice. Les expropriations et les prix des terrains à Paris. 1860-1900. Paris : E. Cornély, 1909.

\section{RÉSUMÉS}

À travers cet entretien, Marcel Roncayolo montre comment les recherches à partir des guides de voyage et de tourisme peuvent contribuer à l'étude de la construction des espaces urbains en Europe. Il explique la légitimité que peut avoir ce type de source pour écrire l'histoire urbaine et pour saisir la complexité d'un territoire. Cet entretien est l'occasion pour Marcel Roncayolo de 
donner sa vision de la géographie et des théories qui la régissent ces derniers temps et d'insister sur sa représentation de la ville à partir de la notion d'itinéraire. Il nous livre aussi son avis sur le découpage territorial.

Through this interview, Marcel Roncayolo shows how researches coming from tourism and traveling guidebooks can contribute to studying the building of urban spaces in Europe. He explains the legitimacy this type of source can get in order to write urban history and catch one territory's complexity. This interview is the opportunity for Marcel Roncayolo to give his own vision of geography and of the theories ruling it since these last years, and to lay stress on his own representation of the city from the notion of route. He reveals as well his opinion on territorial divisions.

\section{INDEX}

Mots-clés : cartographie, découpage territorial, Élisée Reclus, géographie urbaine, guides de voyage, itinéraire, territoires, ville

\section{AUTEUR}

\section{MARCEL RONCAYOLO}

urbaniste et géographe, ancien directeur adjoint de l'École normale supérieure, directeur d'études à l'École des hautes études en sciences sociales, professeur émérite à l'Université Paris-X Nanterre, marcelroncayolo@noos.fr 